\title{
A Simple Confocal Microscopy-based Method for Assessing Sperm Movement
}

\author{
${ }^{\dagger}$ Sung Woo Kim${ }^{1}$, Min Su Kim¹, Chan-Lan Kim¹, In-Sul Hwang² and Ik Soo Jeon ${ }^{1}$ \\ ${ }^{1}$ Animal Genetic Resources Research Center, National Institute of Animal Science, RDA, Namwon 55717, Korea \\ ${ }^{2}$ Animal Biotechnology Division, National Institute of Animal Science, RDA, Wanju 55365, Korea
}

\begin{abstract}
In the field of reproductive medicine, assessment of sperm motility is a key factor for achieving successful artificial insemination, in vitro fertilization, or intracellular sperm injection. In this study, the motility of boar sperms was estimated using real-time imaging via confocal microscopy. To confirm this confocal imaging method, flagellar beats and whiplash-like movement angles were compared between fresh and low-temperature-preserved $\left(17^{\circ} \mathrm{C}\right.$ for $\left.24 \mathrm{~h}\right)$ porcine sperms. Low-temperature preservation reduced the number of flagellar beats from $11.0 \pm 2.3$ beats/s (fresh sperm) to $5.7 \pm 1.8 \mathrm{beats} / \mathrm{s}$ and increased the flagellar bending angle from $19.8^{\circ} \pm 13.8^{\circ}$ (fresh) to $30.6^{\circ} \pm 15.6^{\circ}$. These data suggest that sperm activity can be assessed using confocal microscopy. The observed motility patterns could be used to develop a sperm evaluation index and automated confocal microscopic sperm motility analysis techniques.
\end{abstract}

Key words : Confocal microscope, Sperm motility, Low Temperature preservation

\section{INTRODUCTION}

In animals, the spermatozoa is the most rapidly moving cell in the body; it fertilizes the ovum and exhibits various species-related morphological differences in the head, midpiece, and tail (Phillips, 1972; Suttle et al., 1988; Morales and Overstreet, 1988; Ward, 1998; Calhim et al., 2007; Bonilla and Xu, 2008; Menkveld et al., 2011). The spermatozoa moves via wave-like movements of the midpiece and tail, and this motility is an obvious marker of survival in a fertile environment. Thus, spermatozoa motility and viability analyses provide data regarding sperm quality and infertility risk, and thus, are important for artificial insemination, in vitro fertilization, and assisted reproduction (Foote et al., 1951; Rikmenspoel, 1965; Smith et al.,
1977; Amann, 1989; Vyt et al., 2004).

Sperm motility is currently assessed by visually observing sperms using a microscope. However, this method relies on personal experience and does not present feasible, objective data that could be used by other scientists as a verifiable predictor of the fertilizing potential of spermatozoa (Amann, 1989; Barros et al., 1972; Amelar et al., 1973). Therefore, a computer-assisted semen analyzer (CASA) has been developed to overcome this issue and standardize sperm motility analysis and is considered to be an accurate, rapid, reliable, and objective semen analysis method (Farrel et al., 1998; Coetzee et al., 1999; Amann and Wabersk, 2014). Using CASA, sperm velocity and motility patterns can be accurately analyzed, and automatic assessments of various types of abnormal sperm motility, concentrations,

\footnotetext{
Manuscript received August 29, 2017, Received in revised form September 5, 2017, Accepted September 7, 2017

${ }^{\dagger}$ Corresponding Author: Sung Woo Kim, Ph.D., Animal Genetic Resources Research Center, National Institute of Animal Science, RDA, Namwon 55717, Korea. Tel: +82-63-620-3542, Fax: +82-63-620-3591, E-mail: sungwoo@korea.kr

This is an Open Access article distributed under the terms of the Creative Commons Attribution Non-Commercial License (http:// creativecommons.org/licenses/by-nc/3.0) which permits unrestricted non-commercial use, distribution, and reproduction in any medium, provided the original work is properly cited.
} 
and morphological parameters can be performed. CASA has facilitated the quantification and qualification of various characteristics of semen from many types of animals such as cattle, pigs, poultry, rabbits, dogs, cats, goats, sheep, and humans (Fetterlof and Rogers, 1990; Feldman and Nelson, 1996; Holt et al., 1997; Vyt et al., 2004; Lavara et al., 2005; Betancourt et al., 2006; David et al., 2015). However, despite its usefulness, CASA is expensive and requires an accurate microscope that can distinguish clear contours to identify the sperm head as the center of movement. In this study, we analyzed confocal microscope photos of spermatozoa to develop a simple method of evaluating sperm motility, which is similar to CASA.

Confocal microscopy, frequently called confocal laser scanning microscopy (CLSM), is an imaging technique used for obtaining high optical resolution and contrast via a spatial pinhole placed at the confocal plane of the lens, which eliminates out-of-focus light at specific wavelengths (Patel and McGhee, 2007). CLSM can also generate transmission images by reflecting laser light against an objective sample and converting light photon density into image dot intensity via a digital signal in the targeted area. Image acquisition is based on the principle of scanning; thus, a time difference between the start and end signals of a single image should be manifested. As a result, CLSM images of rapidly moving sperms contain wave-like distortions and bent flagella (including the midpieces and tails). With these data, we realized that CLSM could be used to obtain data regarding sperm motility. In this study, we used simple sperm images obtained using CLSM, rather than the sperm trajectory motility method, to analyze sperm motility and demonstrated flagellar speeds and head movement angles using the real-time mode of CLSM.

\section{MATERIALS \& METHODS}

\section{Animal ethics guideline information}

The protocol and standard operating procedures for the treatment of pigs used in this study were reviewed and approved by the Institutional Animal Care and Use Committee of the National Institute of Animal Science, RDA (Approval number: NIAS2014-489, D-grade).

\section{Semen collection}

Fresh semen was collected from Landrace-strain boars aged 15-36 months using the gloved-hand method. Five sperm samples were subjected to motility testing after dilution in Beltsvile thawing solution (BTS). Four volumes of BTS were slowly added to 1 volume of semen over a 3-5 min period to reduce dilution shock. Diluted samples were then stored in a low-temperature incubator $\left(17^{\circ} \mathrm{C}\right)$.

The cell adhesive CELL-TAK ${ }^{\mathrm{TM}}$ (Becton Dickinson, Franklin Lakes, NJ, USA) was used to fix the sperm heads to the culture dishes as follows. A small volume of adhesive $(2-3 \mu \mathrm{L})$ was applied to the cover glass bottom of confocal dish (SPL Life Science, Gyeonggi-do, Korea). The concentrations of semen dilutions were adjusted to 5-6 $\times$ $10^{6}$ cells $/ \mathrm{ml}$ with BTS, and approximately $50-100 \mu \mathrm{L}$ of each adjusted sample was added to a prepared confocal dish and covered with mineral oil (Sigma, St. Louis, MO, USA). The dish was incubated for $5 \mathrm{~min}$ at $38.5^{\circ} \mathrm{C}$ in a $5 \%$ $\mathrm{CO}_{2}$ incubator, following which unbound sperms were removed by washing with BTS. Finally, sequential images of sperm movement were obtained using a confocal microscope in the real-time mode.

\section{Motility analysis of sperm images}

Five to ten real-time images of live sperm were scanned at 5-s intervals using a confocal microscope with a $40 \times$ objective lens (LSM510, Carl Zeiss, Oberkochen, Germany; FluoView 400, Olympus Corporation, Tokyo, Japan) and one scanning number was used for each images. The $\mathrm{Y}$-axis lengths of the total image and flagellar area were measured according to the scanning times required for image reconstruction using an image analysis program. The flagella imaging time $(x, s)$ could be estimated using 
the proportional expression of $\mathrm{a}(\mu \mathrm{m}): \mathrm{b}(\mu \mathrm{m})=\mathrm{c}(\mathrm{s}): \mathrm{x}(\mathrm{s})$ (Fig. 1A and Table 1). Thus, the flagellar beat speed $(\mathrm{N}$, number $/ \mathrm{s}=\mathrm{n} \times 1 / \mathrm{x})$ and the number of flagellar movements per second could be calculated. To measure the width of the flagellar movement angle in the left-right direction (Fig. 1B), the angles (d) between the extension lines of $\mathrm{a}^{\prime}$ and $\mathrm{c}^{\prime}$ and the extension lines of $\mathrm{b}^{\prime}$ and $\mathrm{d}^{\prime}$ were measured.

\section{Comparison of motility between fresh and low-}

\section{temperature-preserved semen}

To compare differences in sperm motility using CLSM, fresh and low-temperature-preserved $\left(24 \mathrm{~h}\right.$ at $\left.17^{\circ} \mathrm{C}\right)$ semen samples from the same boar were compared.

\section{Statistical analysis}

Each experiment was repeated thrice, and sperm motility data and measured flagellar movement angles were analyzed using Student's $t$-test. An experimental result with a $p$ value of $<0.05$ was considered to be statistically significant.

\section{RESULTS}

\section{Measurement of pig sperm flagellum motility}

The use of real-time CLSM images of living sperm facilitated estimations of sperm speed from the tail trajectory (i.e., wave like) movements. As shown in Fig. 1A, the representative sperm exhibited nine flagellar strokes, leading to a recalculated sperm movement of 14.25 strokes/s; this is shown in Table 1 as an identification number (I.D.) 1 sperm. An analysis of other individual sperms revealed sperm movement ranged from approximately 4 strokes/s (slow moving) to 20 strokes/s (fast moving).

\section{Comparison of sperm flagellar speed between}

\section{fresh and low-temperature-preserved semen}

The speeds of fresh and low-temperature-preserved spermatozoa were determined (Fig. 1A and Fig. 2). When
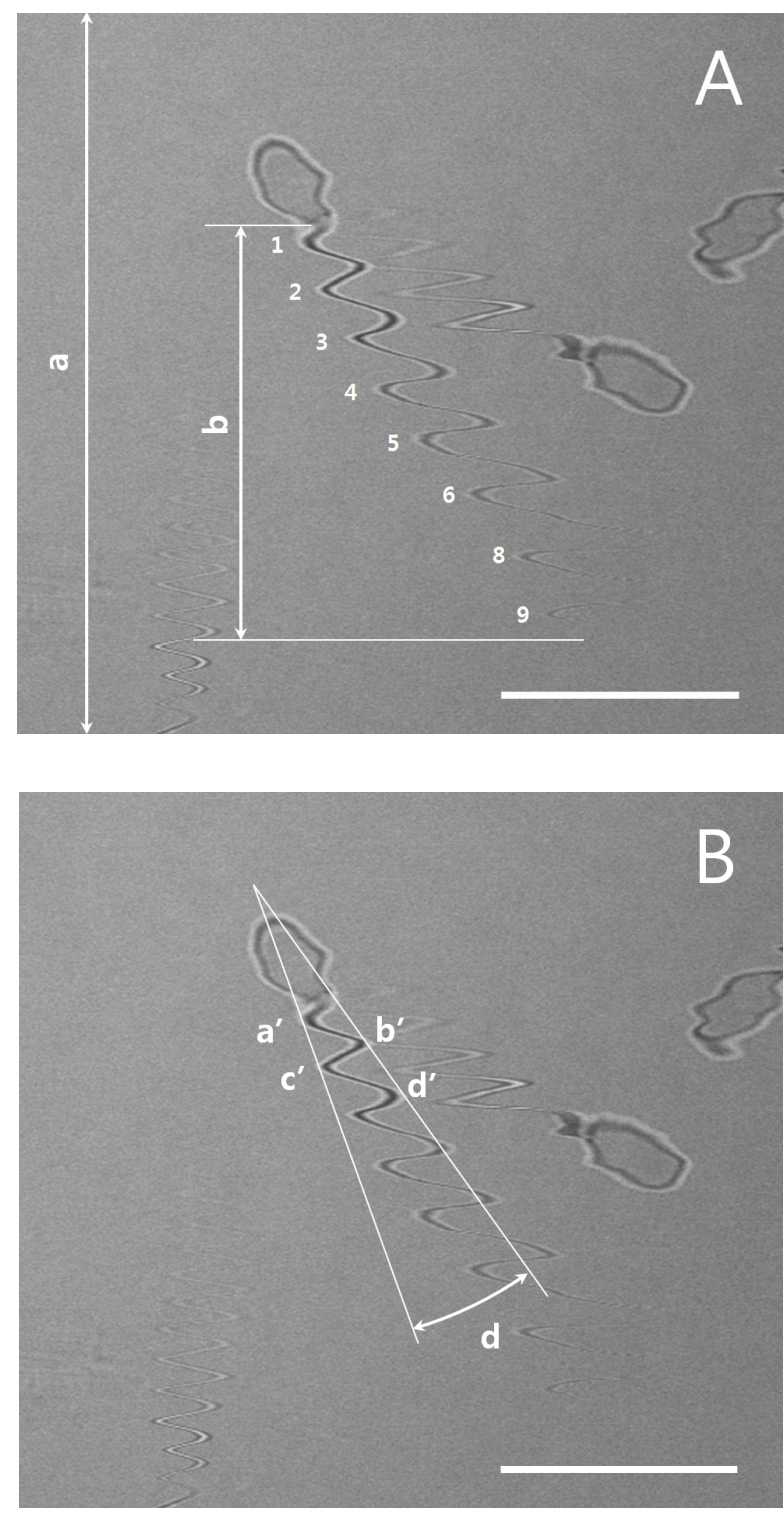

Fig. 1. Confocal laser scanning microscopy (CLSM) imaging analysis of sperm movement. A: The measured $\mathrm{Y}$-axis lengths of the scanned image (a) and sperm flagellum in the beating tail (b) were $60.7 \mu \mathrm{m}$ and $34.8 \mu \mathrm{m}$, respectively. The time taken to scan the total image (c) was measured using a CLSM operating program. As the scanning time of this image was $1.1 \mathrm{~s}$, the scanning time of the flagella was $0.631 \mathrm{~s}(\mathrm{x})$. Sperm tail motility was marked nine times (depicted with numbers). B: The sperm flagellar beating angle formed by the lines of $a^{\prime}-c^{\prime}$ and $b^{\prime}-d^{\prime}$ in the same sperm depicted in A was measured as $15.8^{\circ}$. Scale bar $=20 \mu \mathrm{m}$. 
Table 1. Recalculated measurements of sperm tail motility by confocal imaging

\begin{tabular}{cccc}
\hline \hline Sperm I.D & $\begin{array}{c}\text { Number of flagella motility } \\
\text { in image }(\mathrm{n})\end{array}$ & $\begin{array}{c}\text { The length of Y axis in sperm } \\
\text { movement }(\mathrm{b}, \mu \mathrm{m})\end{array}$ & $\begin{array}{c}\text { Movement of sperm tail } \\
(\mathrm{N}, \text { number/sec) }\end{array}$ \\
\hline 1 & 9 & 34.8 & 14.25 \\
2 & 14 & 38.6 & 20.01 \\
3 & 3 & 40.2 & 4.12 \\
\hline
\end{tabular}

The measurement of the total $\mathrm{Y}$ lengths of the image (a) and sperm tail (b) are illustrated in Fig. 1A. The flagellar activity of the sperm cell with I.D. 1 was recalculated and shown to have normal activity. Representative results from fast-moving (I.D. 2) and slow-moving (I.D. 3) sperms are also shown. I.D., identification number.

porcine semen was preserved at $17^{\circ} \mathrm{C}$ for $24 \mathrm{~h}$, the flagellar speed significantly decreased from $11.0 \pm 2.3$ strokes $/ \mathrm{s}$ (fresh) to $5.7 \pm 1.8$ strokes $/ \mathrm{s}(p<0.05)$.

\section{Comparison of sperm movemenst angles be-} tween fresh and low-temperature-preserved semen

Fig. 3 shows the left-right flagellar movement angles of fresh and low-temperature-preserved spermatozoa using the method described for Fig. 1B. The preserved semen

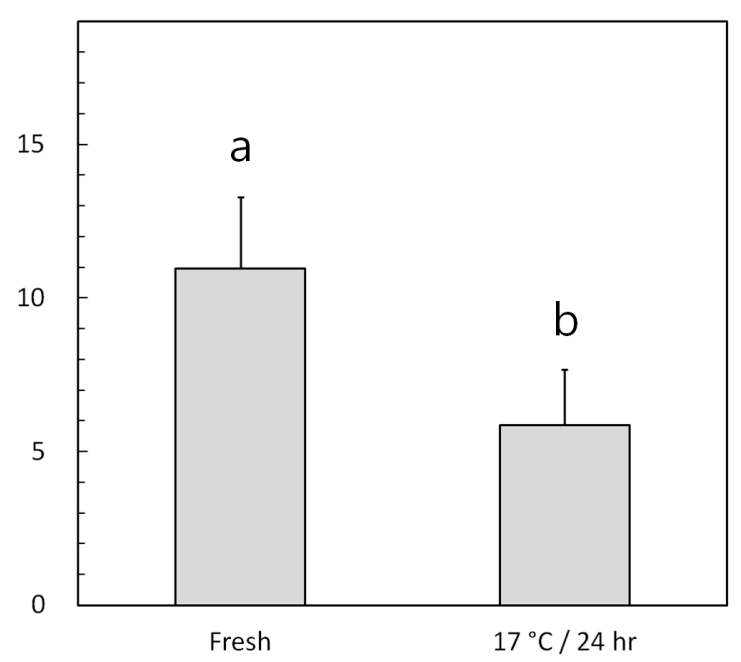

Fig. 2. The effect of low-temperature storage on the flagella beating speed. Eleven and nineteen spermatozoa from fresh and low-temperature-preserved semen, respectively, were analyzed. The experiment was repeated thrice. exhibited a nonsignificant increase in the flagellar movement angle, from $19.8^{\circ} \pm 13.8^{\circ}$ (fresh) to $30.6^{\circ} \pm 15.6^{\circ}$ ( $p>$ $0.05)$.

\section{DISCUSSION}

In this study, we proposed a simple sperm motility analysis method based on flagellar (i.e., sperm tail) speed and movement angle. As mammalian sperms can regulate mo-

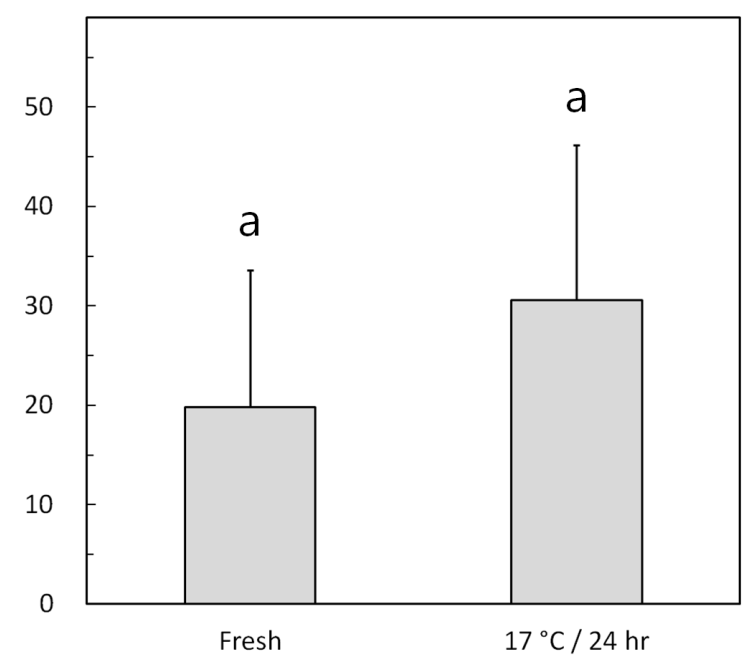

Fig. 3. The effect of low-temperature storage on the flagella beating angle. The spermatozoa described in Fig. 2 were subjected to an analysis of the beating angle as described in Fig. 1B. The experiment was repeated thrice. 
tility and migrate to the locus of fertilization in the female reproductive tract (Eisenbach, 1999; Yoshida and Yashida, 2011), an understanding of individual sperm movement is essential for a sperm quality assessment. Although sperm cells were attached to culture dishes with a cell adhesive, the basic rules of sperm movement would be applied for observing free-moving sperms under a low-magnification objective lens. Conventional semen analysis methods such as CASA track sperm movement by distance or trajectory velocity and the speed of sperm head movement per unit time, thereby automatically calculating the number of spermatozoa in a given field (Amelar et al., 1973; Amann and Wabersk, 2014). Because semen samples contain cells with different morphologies and motility patterns, CASA uses a lower magnitude objective lens $(10 \times$ or $20 \times)$ to observe large numbers of spermatozoa. For real-time analyses, sperms should be treated with stimulants or antagonists to simultaneously yield different data from specific spermatozoa. According to the proposed method, CLSM could be used to image moving sperms; therefore, motility data for individual spermatozoa could be easily obtained by measuring other indicators such as calcium or reactive oxygen species.

Fixation of the sperm heads to the bottoms of culture dishes, as described above, could simulate the event of fertilization, thus demonstrating flagellar movement angles. This technique provided data regarding the distinct behaviors of flagella and the directional movements of cells in contact with a surface. In CASA, sperm tail movement can be expressed as a beat cross frequency (BCF; in $\mathrm{Hz}$ ); except for the lack of attachment, this measurement is similar to our method tested in this study. BCF, which is considered the most constant animal sperm CASA measurement variable, could be used to identify active spermatozoa. Therefore, obtaining data regarding the movements of individual spermatic flagella can facilitate an understanding of sperm physiology by providing a means for experiments regarding individual sperm movement regulation.
This study confirmed that low-temperature preservation reduced sperm motility and affected flagellar activity. Sperm motility is considered an asymmetrical operation that establishes the directionality of movement, a phenomenon that can be easily observed in confocal microscopic images of changes in flagellar movement angles from this and other studies (Rikmenspoel, 1965; Brokaw et al., 1974). In addition, the initial sperm wave motion begins in the neck area and subsequently moves to the tail, and the movement angle determines the directionality of the sperm toward the locus of fertilization by controlling the flagella. We further showed that CLSM imaging of fixed-head spermatozoa could be used for determining the intensity of flagellar movement angles in the left-right direction. The sperm tail movement continuously changes direction to communicate with the ovum via chemotaxis before contact; therefore, flagellar movement angle is an important factor with regard to the mechanism of directionality.

In conclusion, we reported the first simple CLSM-based analysis of sperm movement and directionality. We believe that a related automated analyzing program would accelerate sperm analysis processes.

\section{ACKNOWLEDGEMENTS}

This study was conducted with support from Animal Science \& Technology Development (Project No. PJ010 29304), Rural Development Administration, Republic of Korea. This study was also supported by the 2017 Postdoctoral Fellowship Program of the National Institute of Animal Science, Rural Development Administration, Republic of Korea.

\section{REFERENCES}

Amann RP (1989) Can the fertility potential of a seminal sample be predicted accurately? J Androl 10:89-98.

Amann RP, Wabersk D (2014) Computer-assisted sperm 
analysis (CASA): Capabilities and potential developments. Theriogenology 81:5-17.

Amelar RD, Dubin L, Schoenfeld C (1973) Semen analysis. An office technique. Urology 2:607-611.

Barros C, Fujimoto M, Yanagimachi R (1973) Failure of zona penetration of hamster spermatozoa after prolonged preincubation in a blood serum fraction. J Reprod Fertil 35:89-95.

Betancourt M, Reséndiz A, Fierro ECYR (2006) Effect of two insecticides and two herbicides on the porcine sperm motility patterns using computer-assistedsemen analysis (CASA) in vitro. Reprod Toxicol 22:508-512.

Bonilla E, Xu EY (2008) Identification and characterization of novel mammalian spermatogenic genes conserved from fly to human. Mol Hum Reprod 14:137142.

Brokaw CJ, Josslin R, Bobrow L (1974) Calcium ion regulation of flagellar beat symmetry in reactivated sea urchin spermatozoa. Biochem Biophys Res Commun 58:795-800.

Calhim S, Immler S, Birkhead TR (2007) Postcopulatory sexual selection is associated with reduced variation in sperm morphology. PLoS ONE 2(5):e413.

Coetzee K, Kruger TE, Lombard CJ (1999) Repeatability and variance analysis on multiple computer-assisted (IVOS) sperm morphology readings. Andrologia 31: 163-168.

Cummins JM (1982) Sperm size, body mass, and reproduction in mammals. In: Andre J, editor. The Sperm Cell. The Hague, Martinus Nijholf, pp 395-398.

David I, Kohnke P, Lagriffoul G, Praud O, Plouarboue F, Degond P, Druart X (2015) Mass sperm motility is associated with fertility in sheep. Anim Reprod Sci 161: 75-81.

Eisenbach M (1999) Sperm chemotaxis. Rev Reprod 4:5666.

Farrel PB, Presicce GA, Brockett CC, Foote RH (1998) Quantification of bull sperm characteristics measured by computer-assisted sperm analysis (CASA) and the relationship to fertility. Theriogenology 49:871-879.

Feldmann J, Nelson RW (1996) Canine and feline endocrinology and reproduction. Edited by W.B. Saunders, Philadelphia, pp. 718-733.

Fetterlof PM, Rogers BJ (1990) Prediction of human sperm penetrating ability using computerized motion parameters. Mol Reprod Dev 27:326-331.

Foote RH, Henderson CR, Bratton RW (1951) Some biases to consider in measuring fertility in artificial insemination. J Dairy Sci 34:490.

Holt C, Holt WV, Moore HD, Reed HC, Curnock RM (1997) Objectively measured boar sperm motility parameters correlate with the outcomes of on-farm inseminations: Results of two fertility trials. J Androl 18:312-323.

Immler S, Pryke SR, Birkhead TR, Griffith SC (2010) Pronounced within-individual plasticity in sperm morphometry across social environments. Evolution 64: 1634-1643.

King GJ, Macpherson JW (1973) A comparison of two methods for boar semen collection. J Anim Sci 36:563565 .

Kozdrowski R, Dubiel A, Bielas W, Dzięcioł M (2007) Two protocols of cryopreservation of goat semen with the use of computer-assisted semen analysis system. Acta Veterinaria Brno 76:601-604.

Lavara R. Moce E, Lavara F, De Castro MPV, Vincente JS (2005) Do parameters of seminal quality correlate with the results of on-farm inseminations in rabbits? Theriogenology 64:1130-1141.

Menkveld R, Holleboom CA, Rhemrev JP. (2011) Measurement and significance of sperm morphology. Asian $\mathrm{J}$ Androl 13:59-68.

Morales PK, Overstreet JW (1988) The relationship between the motility and morphology of spermatozoa in human semen. J Androl 9:241-247.

Patel DV, McGhee CN (2007) Contemporary in vivo con- 
focal microscopy of the living human cornea using white light and laser scanning techniques: a major review. Clin Experiment Ophthalmol 35:71-88.

Phillips DM (1972) Comparative analysis of mammalian sperm motility. J Cell Biol 53:561-573.

Rikmenspoel R (1965) The tail movements of bull spermatozoa. Observations and model calculations. Biophys J 5:365-392.

Smith KD, Rodriguez-Rigau LJ, Steinberger E (1977) Relation between indices of sperm analysis and pregnancy rate in infertile couples. Fertil Steril 28:1314-1319.

Suttle JM, Moore HDM, Peirce EJ, Breed WG (1988) Quantitative studies on variation in sperm head morphology of the hopping mouse, Notomys alexis. J Exp
Zool 247:166-171.

Verstegen J, Iguer-Ouada M, Onclin K (2002) Computer assisted semen analyzers in andrology research and veterinary practice. Theriogenology 57:149-179.

Vyt P, Maes D, Rijsselaere T, Dejonckheere E, Castryck F, Soom AV (2004) Motility Assessment of porcine spermatozoa: A comparison of methods. Reprod Dom Anim 39:447-453.

Ward PI (1998) Intraspecific variation in sperm size characters. Heredity 80:655-659.

Yoshida M, Yoshida K (2011) Sperm chemotaxis and regulation of flagellar movement by $\mathrm{Ca}^{2+}$. Mol Hum Repord 17:457-465. 